\title{
A State Duration Model for Brand Choice and Inter-Purchase Time
}

\author{
Lynn $\mathrm{Kuo}^{1}$ and Zhen $\mathrm{Chen}^{2}$ \\ ${ }^{1}$ University of Connecticut and \\ ${ }^{2}$ University of Pennsylvania
}

\begin{abstract}
A new approach for analyzing state duration data in brand-choice studies is explored. This approach not only incorporates the correlation among repeated purchases for a subject, it also models the purchase timing and the brand decision jointly. The former is accomplished by applying transition model approaches from longitudinal studies while the latter is done by conditioning on the brand choice variable. Then mixed multinomial logit models and Cox proportional hazards models are employed to model the marginal densities of the brand choice and the conditional densities of the interpurchase time given the brand choice. We illustrate the approach using a Nielsen household scanner panel data set.
\end{abstract}

Key words: Cox proportional hazards model, longitudinal analysis, multinomial logit model, state duration model.

\section{Introduction}

In brand-choice studies, factors influencing both the brand-switching patterns and purchase-timing decisions are usually of interest. Often, data are collected from $n$ households on their purchase behavior over a period of time. The collected panel data consist of the inter-purchase times and brands chosen, as well as a list of covariates that may include characteristics of the consumers (chooser-specific) and attributes of the brands (choice-specific) at each purchase occasion. For example, the choice-specific explanatory variables may include the price of each brand and an indicator of a special in-store display for each brand; the chooserspecific variables may contain household income and size.

Most of the existing approaches to analyzing these multiple-spell and multipledestination data are restricted to marginal approaches where attention is given either to the state-space part (brand choice) or to the inter-purchase time part. The former includes the discrete brand-choice models of McFadden (1974) and Jain, Vilcassim, and Chintagunta (1994); the latter includes the proportional hazards model (Cox 1972) for the inter-purchase time studied by Jain and Vilcassim (1991). 
The more advanced studies in this area include the conditional model (for the inter-purchase time given the transition states) proposed by Vilcassim and Jain (1991) and Gönül and Srinivasan (1993), and the joint model for the interpurchase time and the brand choice given by Chintagunta and Prasad (1998). Vilcassim and Jain derive a competing risk model where each latent inter-purchase time between transitions is modeled by the Cox regression model with frailty. They found that the probability distribution of inter-purchase times is not the same for various switches between brands. Gönül and Srinivasan (1993) apply a similar approach except that they restrict the slopes to be the same in the Cox regression model for different transitions. Chintagunta and Prasad apply the dynamic McFadden model (Heckman and Singer 1985), where the marginal distribution of the inter-purchase time and the conditional distribution of the brand choice given the inter-purchase time are modeled.

The bivariate model of Chintagunta and Prasad provides further advancement in the area. However, improvement can be made upon their models. First, they fit the same model for the inter-purchase time independent of the transitional states. This is unsatisfactory according to the findings of Vilcassim and Jain, because the probability distribution of inter-purchase times is usually not the same for various switches between brands. Second, they assume the same model and conditional independence for the multiple spells within each household and between households. These assumptions are also too restrictive, because multiple spells within each household tend to be much more similar than between households.

In this paper, we propose an alternative bivariate model (a composite transition model) for analyzing the multiple-spell and multiple-destination data. Our new method not only treats both the inter-purchase times and the brand choice as random outcomes but also incorporates the dependence between successive spells for the same household. First we use the transition model approach (Diggle, Liang, and Zeger 1994, pp. 192-193) in longitudinal studies to develop the likelihood as a product of conditional bivariate densities of the response in each spell given the previous spell. Then we rewrite each of the conditional bivariate densities as a product of two densities by conditioning on the brand choice variable as opposed to conditioning on the purchase timing variable as in the dynamic McFadden model. We use a mixed multinomial logit model for the first density (the discrete choice transition part) and the Cox regression model for the second density (the conditional density of the inter-purchase time given the two sandwiching brand choices).

Although our discussion in this paper is focused on brand-choice studies, our methodology is general and can be applied to many state duration data. Our method has several advantages over the existing approaches for analyzing 
multiple-spell and multiple-destination data. (1) We jointly model the bivariate outcomes of the brand choice and inter-purchase time; thus the model is more complete than the marginal model for either variable or the conditional model discussed earlier. (2) Our model incorporates a dependence structure across successive purchases, thus providing a more realistic framework in modeling the multiple-spell data. (3) Our model links many existing methods, for example, the marginal method for discrete choice and the conditional method by Vilcassim and Jain. From the next section where detailed derivation for our likelihood is given, we see the conditional model of Vilcassim and Jain is a component of our composite model. Therefore, we are providing a theoretical justification for the conditional transition model given by Vilcassim and Jain. Consequently, our derivation helps to explain and clarify the roles that some of the existing methods play in brand-choice studies. (4) Our model has an easy interpretation where each component of the composite model (either discrete choice model or duration model) is well understood. (5) The inter-purchase time part of our composite model is modeled on the minimum of the latent transition times. In this respect, it is simpler than that of Vilcassim and Jain. It also avoids the pitfalls of the competing risk models (cf. Prentice, Kalbfleisch, Peterson, Flounoy, Farewell, and Breslow, 1978). Finally, (6) Our model is easy to fit, requiring a straightforward manipulation of the data set and applying existing software (i.e., SAS or Splus). We can fit the data for the discrete choice model separately from the Cox regression model. The former requires that we divide the data into $L$ (the number of brands) groups; each group consists of pairs of consecutive brand choices with the same past brand. Then we fit a mixed multinomial logit model to the present brand choices for each group. The latter requires that we fit separate Cox regression models for each of the subgroups of the inter-purchase times with the same sandwiching transition states. This can be done easily by many software. For example, we can use the procedure PHREG in SAS with a BY statement.

We apply our new method to the Nielsen household scanner panel data set that was previously analyzed by Chintagunta and Prasad (1998). In the analysis, we use essentially the same predictors (price, feature, household size, previous volume, and non-detergent expenditure) that they used except we add the household size (chooser-specific) in the discrete choice model. We found that not only price and feature advertisement are significant among all the choice-specific variables, but also household size is significant among all chooser-specific variables. The effects of these predictors are generally in agreement with that in Chintagunta and Prasad. For example, from the brand choice study, reduced price and feature advertisement enhance purchasing.

We organize the rest of the paper as follows. Section 2 outlines the model 
formulation and discusses the discrete choice model and the duration model that are integral parts of the composite model. Section 3 discusses the estimation method. Section 4 presents the data and some numerical results, and Section 5 summarizes our study and remarks on future research directions.

\section{Model Formulation}

\subsection{Transition model}

Suppose that we have $n$ households in the brand choice study, and that the $i$ th household makes a total of $n_{i}$ repeated purchases in a fixed time period. We record the purchase timing and the brand choice at each purchase. It is assumed that each household is allowed to choose only one of the $L$ brands for each purchase. Let $w_{i j}$ denote the observed calendar time of the $j$ th purchase of the $i$ th household and let $y_{i j}$ denote the brand chosen by the household at this epoch. We set $y_{i j}$ to be a discrete index variable with integer values ranging from 1 to $L$. Then we can summarize our data for the $i$ th household by a series of bivariate observations $\left\{\left(t_{i j}, y_{i j}\right)\right\}$, with $j=1, \ldots, n_{i}$, where $t_{i j}=w_{i j}-w_{i, j-1}$, the $j$ th inter-purchase time for the $i$ th household. We define $w_{i 0}=0$. Along with these bivariate outcomes, we also observe a vector of chooser-specific variables $\boldsymbol{x}_{i j}$ and a vector of choice-specific variables $\boldsymbol{z}_{i j}$, for the $j$ th purchase of the $i$ th household, where $\boldsymbol{z}_{i j}=\left(\boldsymbol{z}_{i j 1}^{\prime}, \ldots, \boldsymbol{z}_{i j L}^{\prime}\right)^{\prime}$, with $\boldsymbol{z}_{i j l}$ itself a column vector of choicespecific variables for the $l$ th brand. Choice-specific variables usually change over time. Some of the chooser-specific variables take the same value across the spells for each consumer; nevertheless, we do allow them to be time-variant covariates.

Generally, the $n_{i}$ bivariate outcomes, $\left(t_{i 1}, y_{i 1}\right), \ldots,\left(t_{i n_{i}}, y_{i n_{i}}\right)$, from the $i$ th household exhibit correlations, because the past values $\left\{\left(t_{i 1}, y_{i 1}\right), \ldots,\left(t_{i, j-1}, y_{i, j-1}\right)\right\}$ influence the present observation $\left(t_{i j}, y_{i j}\right)$. For brevity, we define the history for the $i$ th household at the $j$ th purchase to be $H_{i j}=\left\{\left(t_{i k}, y_{i k}\right), k=1, \ldots, j-1\right\}$. Therefore the joint density of the $n_{i}$ outcomes for the $i$ th household is given by

$$
f_{i}\left(\left(t_{i 1}, y_{i 1}\right), \ldots,\left(t_{i n_{i}}, y_{i n_{i}}\right)\right)=f\left(t_{i 1}, y_{i 1}\right) \prod_{j=2}^{n_{i}} f\left(t_{i j}, y_{i j} \mid H_{i j}\right) .
$$

All the densities here are also functions of the covariates $\boldsymbol{x}$ and $\boldsymbol{z}$. We suppress them in all the equations in this subsection for simpler notation.

Suppose we are willing to accept a first-order Markov property. Then the above formula (the $i$ th subject's contribution to the likelihood) can be simplified to:

$$
f_{i}\left(\left(t_{i 1}, y_{i 1}\right), \ldots,\left(t_{i n_{i}}, y_{i n_{i}}\right)\right)=f\left(t_{i 1}, y_{i 1}\right) \prod_{j=2}^{n_{i}} f\left(t_{i j}, y_{i j} \mid t_{i, j-1}, y_{i, j-1}\right)
$$


For the joint model $f\left(t_{i j}, y_{i j} \mid t_{i, j-1}, y_{i, j-1}\right)$, we decompose it further into a composite model by conditioning on the $y_{i j}$ variable; that is we write the joint density as a product of the marginal density of $y_{i j}$ and a conditional density for $t_{i j}$ given $y_{i j}$, both conditioning on $\left(t_{i, j-1}, y_{i, j-1}\right)$. Therefore, we have

$$
f\left(t_{i j}, y_{i j} \mid t_{i, j-1}, y_{i, j-1}\right)=f\left(y_{i j} \mid t_{i, j-1}, y_{i, j-1}\right) f\left(t_{i j} \mid y_{i j}, t_{i, j-1}, y_{i, j-1}\right) .
$$

Then we will make the following two assumptions in modeling the composite model. First,

$$
f\left(y_{i j} \mid t_{i, j-1}, y_{i, j-1}\right)=f\left(y_{i j} \mid y_{i, j-1}\right) .
$$

This assumes that $y_{i j}$ is independent of $t_{i, j-1}$ conditioning on $y_{i, j-1}$. It assumes that once we know the previous brand choice, the previous inter-purchase time carries no information about the current brand choice. This makes intuitive sense.

Second, we assume

$$
f\left(t_{i j} \mid y_{i j}, t_{i, j-1}, y_{i, j-1}\right)=f\left(t_{i j} \mid y_{i, j-1}, y_{i j}\right)
$$

This assumes that the two transition states sandwiching the inter-purchase time are sufficient to determine the inter-purchase time. That is, the inter-purchase time is independent of the previous inter-purchase time given the two sandwiching states.

We note that the first-order Markov property and the above two assumptions are equivalent to a semi-Markov assumption on the sequence $\left\{\left(t_{i j}, y_{i j}\right), j=\right.$ $1,2, \ldots)\}$ (see, for example, Lawless and Fong 1999).

Substituting the above two assumptions (2.3) and (2.4) into the composite model (2.2), we derive the likelihood function for all the households from (2.1):

$$
\prod_{i=1}^{n} f_{i}\left(\left(t_{i 1}, y_{i 1}\right), \ldots,\left(t_{i n_{i}}, y_{i n_{i}}\right)\right)=\prod_{i=1}^{n} \prod_{j=1}^{n_{i}} f\left(y_{i j} \mid y_{i, j-1}\right) f\left(t_{i j} \mid y_{i, j-1}, y_{i j}\right)
$$

where $f\left(y_{i 1} \mid y_{i 0}\right)=f\left(y_{i 1}\right)$ and $f\left(t_{i 1} \mid y_{i 0}, y_{i 1}\right)=f\left(t_{i 1} \mid y_{i 1}\right)$. This suggests that we need to model the first-order discrete choice transitions and to model the duration given the transition states. Both can be done based on the existing literature.

It can be argued that the assumption 2 in (2.4) is too strong, because the previous inter-purchase times are expected to carry information about the present inter-purchase times. We will consider an additional analysis without the second assumption. In this respect, to model $f\left(t_{i j} \mid y_{i j}, t_{i, j-1}, y_{i, j-1}\right)$, we still keep the same structure of modeling the duration given the two sandwiching transition states except we add the previous inter-purchase time as a covariate in the duration model. 


\subsection{Discrete choice model}

Let us first discuss the discrete choice part. Given that the previous choice only takes on one of the $L$ values, we consider a class of models conditional on the previous choice.

Suppose $\boldsymbol{x}_{i j}=\left(1, x_{i j 1}, \ldots, x_{i j k}\right)^{\prime}$ is a set of chooser specific covariates for the $j$ th purchase of the $i$ th household. Let us assume the previous choice to be $l$. Let $\boldsymbol{\alpha}_{m}^{(l)}$ denote a $k+1$ dimension column vector. We use the notation $\pi_{i j}(m \mid l)$ to denote $f\left(y_{i j}=m \mid y_{i, j-1}=l\right)$. Then the generalized logit model (Agresti 1990, Hosmer and Lemeshow 1989, and McCullagh and Nelder 1989) suggests

$$
\pi_{i j}(m \mid l)=\frac{\exp \left(\boldsymbol{\alpha}_{m}^{(l)^{\prime}} \boldsymbol{x}_{i j}\right)}{\sum_{m=1}^{L} \exp \left(\boldsymbol{\alpha}_{m}^{(l)^{\prime}} \boldsymbol{x}_{i j}\right)} .
$$

We may set $\boldsymbol{\alpha}_{m}^{(l)}=(0, \ldots, 0)^{\prime}$ for $m=l$ in the above model for identifiability purpose. The log odds ratio for choosing $m$ over $n$ is

$$
\log \left(\frac{\pi_{i j}(m \mid l)}{\pi_{i j}(n \mid l)}\right)=\left(\boldsymbol{\alpha}_{m}^{(l)}-\boldsymbol{\alpha}_{n}^{(l)}\right)^{\prime} \boldsymbol{x}_{i j}
$$

Let $\boldsymbol{z}_{i j m}$ be a column vector of choice-specific variables associated with the brand choice $m$ for the $j$ th purchase of the $i$ th household. Let $\boldsymbol{\beta}^{(l)}$ denote a column vector with the same dimension as $\boldsymbol{z}_{i j m}$. To model the transition discrete choice probabilities, we can consider the multinomial logit model (McFadden 1974). That is,

$$
\pi_{i j}(m \mid l)=\frac{\exp \left(\boldsymbol{\beta}^{(l)^{\prime}} \boldsymbol{z}_{i j m}\right)}{\sum_{m=1}^{L} \exp \left(\boldsymbol{\beta}^{(l)^{\prime}} \mathbf{z}_{i j m}\right)} .
$$

Then

$$
\log \left(\frac{\pi_{i j}(m \mid l)}{\pi_{i j}(n \mid l)}\right)=\boldsymbol{\beta}^{(l)^{\prime}}\left(\boldsymbol{z}_{i j m}-\boldsymbol{z}_{i j n}\right) .
$$

This model implies the log odds of choosing $m$ over $n$ depend on the distance between the values of the variables of the two brands in the comparison. If the values of a particular variable for both brands are the same, the model asserts that this variable has no influence on the choice between the alternatives $m$ and $n$.

We can also formulate a mixed multinomial logit model containing both chooser-specific and choice-specific covariates (cf. Agresti 1990). For example, we define

$$
\boldsymbol{x}_{i j m}^{*}=\left(\boldsymbol{z}_{i j m}^{\prime}, \delta_{m 1} \boldsymbol{x}_{i j}^{\prime}, \ldots, \delta_{m L} \boldsymbol{x}_{i j}^{\prime}\right)^{\prime}, \quad m=1, \ldots, L,
$$


where $\delta_{m t}$ equals 1 when $t=m$ and 0 otherwise, and $\boldsymbol{z}_{i j m}$ and $\boldsymbol{x}_{i j}$ are defined as before. Let $\boldsymbol{\beta}^{*(l)}=\left(\boldsymbol{\beta}^{(l)^{\prime}}, \boldsymbol{\alpha}_{1}^{(l)^{\prime}}, \ldots, \boldsymbol{\alpha}_{L}^{(l)^{\prime}}\right)^{\prime}$. Then $\boldsymbol{\beta}^{*(l)^{\prime}} \boldsymbol{x}_{i j m}^{*}=\boldsymbol{\beta}^{(l)^{\prime}} \boldsymbol{z}_{i j m}+$ $\boldsymbol{\alpha}_{m}^{(l)^{\prime}} \boldsymbol{x}_{i j}$. Thus the mixed multinomial logit model containing both choice-specific and chooser-specific variables is

$$
\begin{aligned}
\pi_{i j}(m \mid l) & =\frac{\exp \left(\boldsymbol{\beta}^{(l)^{\prime}} \mathbf{z}_{i j m}+\boldsymbol{\alpha}_{m}^{(l)^{\prime}} \boldsymbol{x}_{i j}\right)}{\sum_{m=1}^{L} \exp \left(\boldsymbol{\beta}^{(l)^{\prime}} \boldsymbol{z}_{i j m}+\boldsymbol{\alpha}_{m}^{(l)^{\prime}} \boldsymbol{x}_{i j}\right)} \\
& =\frac{\exp \left(\boldsymbol{\beta}^{*(l)^{\prime}} \boldsymbol{x}_{i j m}^{*}\right)}{\sum_{m=1}^{L} \exp \left(\boldsymbol{\beta}^{*(l)^{\prime}} \boldsymbol{x}_{i j m}^{*}\right)},
\end{aligned}
$$

since, for identifiability, we can take $\boldsymbol{\alpha}_{m}^{(l)}=(0, \ldots, 0)^{\prime}$ for $m=l$. Then the log odds of choosing $m$ over $n$ becomes

$$
\begin{aligned}
\log \left(\frac{\pi_{i j}(m \mid l)}{\pi_{i j}(n \mid l)}\right) & =\boldsymbol{\beta}^{*(l)^{\prime}}\left(\boldsymbol{x}_{i j m}^{*}-\boldsymbol{x}_{i j n}^{*}\right) \\
& =\boldsymbol{\beta}^{(l)^{\prime}}\left(\boldsymbol{z}_{i j m}-\boldsymbol{z}_{i j n}\right)+\left(\boldsymbol{\alpha}_{m}^{(l)^{\prime}}-\boldsymbol{\alpha}_{n}^{(l)^{\prime}}\right) \boldsymbol{x}_{i j} .
\end{aligned}
$$

The mixed multinomial logit model in (2.6) enables us to incorporate consumer characteristics, intercept, and other choice-specific variables in the McFadden model. It shares the same desirable interpretations as the generalized logit model and the McFadden model discussed earlier.

\subsection{Duration model}

For the conditional model of the inter-purchase time given the sandwiching brand choices, we apply standard models in duration analysis (Kalbfleisch and Prentice 1980). In particular, let $h\left(t_{i, j} \mid y_{i, j-1}=l, y_{i j}=m\right)$ denote the hazard function of $t_{i j}$ conditional on the two sandwiching brand choices. Then, we consider the proportional hazards model

$$
h\left(t_{i j} \mid y_{i, j-1}=l, y_{i j}=m\right)=h_{0}^{(l m)}(t) \exp \left(\boldsymbol{\lambda}^{(l m)^{\prime}} \boldsymbol{x}_{i j}+\boldsymbol{\xi}^{(l m)^{\prime}} \boldsymbol{z}_{i j}\right)
$$

where $l, m=1, \ldots, L, \boldsymbol{\lambda}^{(l m)}$ is a column vector of the same length as $\boldsymbol{x}_{i j}$ (the chooser-specific variable for the $j$ th purchase of the $i$ th household), $\boldsymbol{\xi}^{(l m)}$ is a column vector of the same length as $\boldsymbol{z}_{i j}$ (the choice-specific variable for the same purchase), and $h_{0}^{(l m)}(t)$ is the baseline hazard function for the state $l$ to state $m$ transition. Additional, we consider the model with the previous inter-purchase time added as part of the covariates to relax the assumption 2 in (2.4) we made earlier. 
Therefore, the conditional density $f\left(t_{i j} \mid y_{i, j-1}=l, y_{i j}=m\right)$ is modeled by

$$
\begin{aligned}
& f\left(t_{i j} \mid y_{i, j-1}=l, y_{i j}=m\right) \\
= & h\left(t_{i j} \mid y_{i, j-1}=l, y_{i j}=m\right) \exp \left\{-\int_{0}^{t_{i j}} h\left(u \mid y_{i, j-1}=l, y_{i j}=m\right) d u\right\} .
\end{aligned}
$$

Flinn and Heckman $(1982,1983)$ suggest using the Box-Cox formulation to specify the baseline hazard $h_{0}^{(l m)}(t)$. That is,

$$
h_{0}(t)=\exp \left(\gamma_{0}+\sum_{k=1}^{K} \gamma_{k}\left(\frac{t^{\lambda_{k}}-1}{\lambda_{k}}\right)\right)
$$

where $\gamma_{0}, \lambda_{k}$ and $\gamma_{k}, k=1, \ldots, K$ are parameters to be estimated. These parameters depend on the inter-purchase times between $l$ to $m$ transitions. We omit the superscripts $(\mathrm{lm})$ for simplicity. One advantage of this representation of the baseline hazard is that many frequently used probability distributions for durations are nested within it. For example, if $\gamma_{k}=0$ for all $k \geq 1$, then $h_{0}(t)=\exp \left(\gamma_{0}\right)$, hence the baseline hazard corresponds to an exponential distribution. Weibull and extreme value distributions are also nested within (2.8). Thus this formula enables researchers to test the adequacy of a particular distribution for the duration.

On the other hand, studies (for example, Jain and Vilcassim 1991) have shown that in marketing the duration time may not be able to be characterized by those common distributions nested within the Box-Cox formulation. Moreover, some common distributions are not nested in the formula. For example, lognormal and logistic distributions are not in this family. Thus the use of the Box-Cox representation is quite limited. Therefore we leave the baseline hazard totally arbitrary. The resulted model is a semiparametric Cox regression model. This model assumes that the hazard ratio (relative risk) for two inter-purchase times with the same sandwiching states is a constant (with respect to $t$ ).

\section{Estimation}

Let us recall that the likelihood in $(2.5)$ can be written as a product of two parts

$$
L=\left\{\prod_{i=1}^{n} \prod_{j=1}^{n_{i}} f\left(y_{i j} \mid y_{i, j-1}\right)\right\}\left\{\prod_{i=1}^{n} \prod_{j=1}^{n_{i}} f\left(t_{i j} \mid y_{i, j-1}, y_{i j}\right)\right\},
$$

where we use the mixed multinomial logit models (2.6) for the first part (the discrete choice transition model) and the Cox proportional hazards models (2.7) for the second part (the conditional densities for the inter-purchase times given 
the two sandwiching states). To maximize this likelihood, it suffices to maximize each part separately.

Define $\mathcal{H}^{(l)}=\left\{(i, j) \mid y_{i, j-1}=l\right\}$. It is the subset of double indices on $i$ and $j$ for the $i^{\text {th }}$ household with the $j$ th purchase such that $y_{i, j-1}=l$. Then we can rewrite the first part of the likelihood in (3.1) as

$$
L_{p}=\prod_{i=1}^{n} \prod_{j=1}^{n_{i}} f\left(y_{i j} \mid y_{i, j-1}\right)=\prod_{l=1}^{L}\left\{\prod_{(i, j) \in \mathcal{H}^{(l)}} f\left(y_{i j} \mid y_{i, j-1}=l\right)\right\} .
$$

Recall our mixed multinomial logit model is defined by

$$
f\left(y_{i j}=m \mid y_{i, j-1}=l\right)=\pi_{i j}(m \mid l)=\frac{\exp \left(\boldsymbol{\beta}^{*(l)^{\prime}} \boldsymbol{x}_{i j m}^{*}\right)}{\sum_{m=1}^{L} \exp \left(\boldsymbol{\beta}^{*(l)^{\prime}} \boldsymbol{x}_{i j m}^{*}\right)} .
$$

Therefore, the partial likelihood $L_{p}$ can be rewritten as

$$
\begin{aligned}
L_{p} & =\prod_{l=1}^{L}\left\{\prod_{(i, j) \in \mathcal{H}^{(l)}} \prod_{m=1}^{L} f\left(y_{i j}=m \mid y_{i, j-1}=l\right)^{I\left(y_{i j}=m\right)}\right\} \\
& =\prod_{l=1}^{L}\left\{\prod_{(i, j) \in \mathcal{H}^{(l)}} \frac{\exp \left(\boldsymbol{\beta}^{*(l)^{\prime}} \sum_{m=1}^{L} \delta_{i j m} \boldsymbol{x}_{i j m}^{*}\right)}{\sum_{m=1}^{L} \exp \left(\boldsymbol{\beta}^{*(l)^{\prime}} \boldsymbol{x}_{i j m}^{*}\right)}\right\} \\
& =\prod_{l=1}^{L} L_{l}\left(\boldsymbol{\beta}^{*(l)}\right),
\end{aligned}
$$

where $\delta_{i j m}=I\left(y_{i j}=m\right)$.

Therefore, to maximize the likelihood of the discrete choice transition part, it suffices to fit each of the $L$ mixed multinomial logit models separately, each with only transition pairs with the same brand choice of last purchase. Then we fit the mixed multinomial logit model to the present brand choice. The NewtonRaphson method can be used to maximize the likelihood $L_{l}\left(\boldsymbol{\beta}^{*(l)}\right)$. Nevertheless, we can find the MLE easily by fitting a stratified proportional hazards model (cf. Klein and Moeschberger 1997, p. 282). For example, it can be done with the procedure PHREG with the strata option in SAS. Observe for each subject in $\mathcal{H}^{(l)}$, we can create $L$ dummy survival times with the $m$ th subject's survival time 0 , if $y_{i j}=m$ and the remaining $L-1$ subjects censored at a later time (say 1). Moreover, the covariates for the $m$ th subject are $\boldsymbol{x}_{i j m}^{*}$. Then the partial likelihood employed in the Cox regression model is exactly

$$
\frac{\exp \left(\boldsymbol{\beta}^{*(l)^{\prime}} \sum_{m=1}^{L} \delta_{i j m} \boldsymbol{x}_{i j m}^{*}\right)}{\sum_{m=1}^{L} \exp \left(\boldsymbol{\beta}^{*(l)^{\prime}} \boldsymbol{x}_{i j m}^{*}\right)}
$$


because the risk set for the $m$ th subjects includes all the $L$ subjects. Then we add a strata option by ID for all subjects in $\mathcal{H}^{(l)}$. Essentially, the strata option treats each set of the $L$ dummy survival times just created as a stratum. This will allow us to maximize $L_{l}\left(\boldsymbol{\beta}^{*(l)}\right)$. See SAS (1995 pp. 131-144) for more details.

We apply a similar idea to fit the duration model except we need to divide our likelihood into a product of $L \times L$ terms. Recall that we use the same Cox regression model for the inter-purchase times with the same sandwiching states, for example, for all the consecutive states with $y_{i, j-1}=l$ and $y_{i j}=m$. Therefore, to maximize the likelihood for the inter-purchase time part, it suffices to fit $L^{2}$ models separately, where each model (say the $(l m)$ th model) only picks up the inter-purchase times with the $l$ to $m$ transition. It is relatively easy to use existing software to fit separate Cox regression models for each transition to obtain the estimates of the regression coefficients and the baseline hazard function for each transition. The regression coefficients estimates are actually the maximum likelihood estimates for the partial likelihood usually employed in the Cox model that ignores the baseline hazard function. Then the baseline hazard function is estimated nonparametrically by the Breslow (1974) method.

\section{Data and Results}

We reanalyze the Nielsen household scanner panel data set on purchases of liquid laundry detergents in Sioux Falls, South Dakota, that was recently evaluated by Chintagunta and Prasad (1998). The observation period for this data lasted from the first week of July, 1986 to July 16, 1988, a two year span. The sample consists of 400 households who purchased only from among six national brands, Tide, EraPlus, Solo, Wisk, Surf, and All. From these households, we collected a total of 3,055 spells. For scanner data, the first and the last spells are usually incomplete. The inter-purchase time of the first spell is left-truncated, and thus we also don't know the starting brand for the first spell. The last spell is usually censored at the end of the study; therefore we only know that the interpurchase time should be larger than the time observed. As a consequence, we don't know what brand the household will choose at the end of the last spell. For our data, all 400 households have incomplete first spells, and 398 households have incomplete last spells. Many of the incomplete spells have missing covariates.

Special methods are needed for handling these two special situations. In this paper, however, we will simply drop these incomplete spells. The resulted complete sample consists of 320 households and 2,257 spells. Of the 320 households, most of them $(72 \%)$ have less than ten spells, about $50 \%$ have six or less spells, $90 \%$ have eighteen of less spells, eight (2.5\%) have more than 30 spells, two have more than 40 spells, and one has 87 spells.

Table 1 tabulates the frequency counts of the $l$ to $m$ transitions for each of 
Table 1: Transition matrix

\begin{tabular}{|c|c|c|c|c|c|c|c|}
\hline & \multicolumn{6}{|c|}{ To } & \multirow{2}{*}{$\begin{array}{l}\text { Row } \\
\text { Total }\end{array}$} \\
\hline & TIDE & WISK & ERAPLUS & SURF & SOLO & ALL & \\
\hline \multirow[t]{2}{*}{ TIDE } & $415(18.4)$ & $\overline{59(2.6)}$ & $40(1.8)$ & $\overline{\mathbf{5 2}(2.3)}$ & $12(0.5)$ & $\mathbf{1 0}(0.4)$ & $588(26.1)$ \\
\hline & $70.6 / 70.3$ & $10.0 / 10.0$ & $6.8 / 6.8$ & $8.8 / 8.8$ & $2.0 / 2.0$ & $1.7 / 1.7$ & \\
\hline \multirow[t]{2}{*}{ WISK } & $67(3.0)$ & $461(20.4)$ & $37(1.6)$ & $\mathbf{2 8}(1.2)$ & $8(0.4)$ & $3(0.1)$ & $604(26.8)$ \\
\hline & $11.1 / 11.1$ & $76.3 / 76.2$ & $6.8 / 6.1$ & $4.6 / 4.6$ & $1.3 / 1.3$ & $0.5 / 0.5$ & \\
\hline \multirow[t]{2}{*}{ ERAPLUS } & $\mathbf{3 7}(1.6)$ & $32(1.4)$ & $320(14.2)$ & 33 (1.5) & $12(0.5)$ & $\mathbf{0}(0.0)$ & $434(19.2)$ \\
\hline & $8.5 / 8.4$ & $7.4 / 7.3$ & $73.7 / 72.9$ & $7.6 / 7.5$ & $2.8 / 2.7$ & $0.0 / 0.0$ & \\
\hline \multirow{2}{*}{ SURF } & $56(2.5)$ & $43(1.9)$ & $\mathbf{2 6}(1.2)$ & $196(8.7)$ & $14(0.6)$ & $4(0.2)$ & $339(15.0)$ \\
\hline & $16.5 / 16.9$ & $12.7 / 13.0$ & $7.7 / 7.8$ & $57.8 / 59.0$ & $4.1 / 4 / 2$ & $1.2 / 1.2$ & \\
\hline \multirow[t]{2}{*}{ SOLO } & $\mathbf{1 1}(0.5)$ & $\mathbf{5}(0.2)$ & $12(0.5)$ & $21(0.9)$ & $171(7.6)$ & $1(0.0)$ & $221(9.8)$ \\
\hline & $5.0 / 5.0$ & $2.3 / 2.3$ & $5.4 / 5.5$ & $9.5 / 9.6$ & $77.4 / 78.1$ & $0.5 / 0.5$ & \\
\hline \multirow[t]{2}{*}{ ALL } & $4(0.2)$ & $5(0.2)$ & $4(0.2)$ & $2(0.1)$ & $2(0.1)$ & $\mathbf{5 4}(2.4)$ & 71 (3.1) \\
\hline & $6.6 / 5.6$ & $7.0 / 6.9$ & $5.6 / 5.6$ & $2.8 / 2.8$ & $2.8 / 2.8$ & $76.1 / 75.0$ & \\
\hline Col Total & $590(26.1)$ & $605(26.8)$ & $439(19.5)$ & $332(14.7)$ & $219(9.7)$ & $72(3.2)$ & 2257 \\
\hline
\end{tabular}

The four entries in each cell are frequency [in bold face], total frequencey [in ( ) ], and row point/column point.

the $6 \times 6$ transitions among the 2,257 spells. This table suggests the degree of competition among the brands, without controlling for the covariates. The large magnitude of the diagonal elements relative to the off diagonal ones suggests the tendency of the consumers to keep buying the same brand. In fact, almost three quarters $(71 \%)$ of the transitions are repeated purchases of the same brand. Table 2 summarizes the mean inter-purchase time for each transition. The mean inter-purchase time for the whole sample is about 55 days.

Table 2: Average interpurchase time (in days) by transitions

\begin{tabular}{cccccccc}
\hline & \multicolumn{9}{c}{ To } & & Row \\
From & TIDE & WISK & ERAPLUS & SURF & SOLO & ALL & Average \\
\hline TIDE & 49 & 61 & 67 & 65 & 117 & 74 & 54 \\
WISK & 75 & 52 & 71 & 70 & 70 & 146 & 57 \\
ERAPLUS & 54 & 55 & 53 & 44 & 46 & - & 53 \\
SURF & 68 & 81 & 59 & 45 & 82 & 55 & 56 \\
SOLO & 64 & 56 & 48 & 71 & 46 & 123 & 50 \\
ALL & 92 & 66 & 198 & 51 & 48 & 62 & 71 \\
\hline Column Average & 54 & 55 & 58 & 52 & 53 & 67 & 55 \\
\hline
\end{tabular}




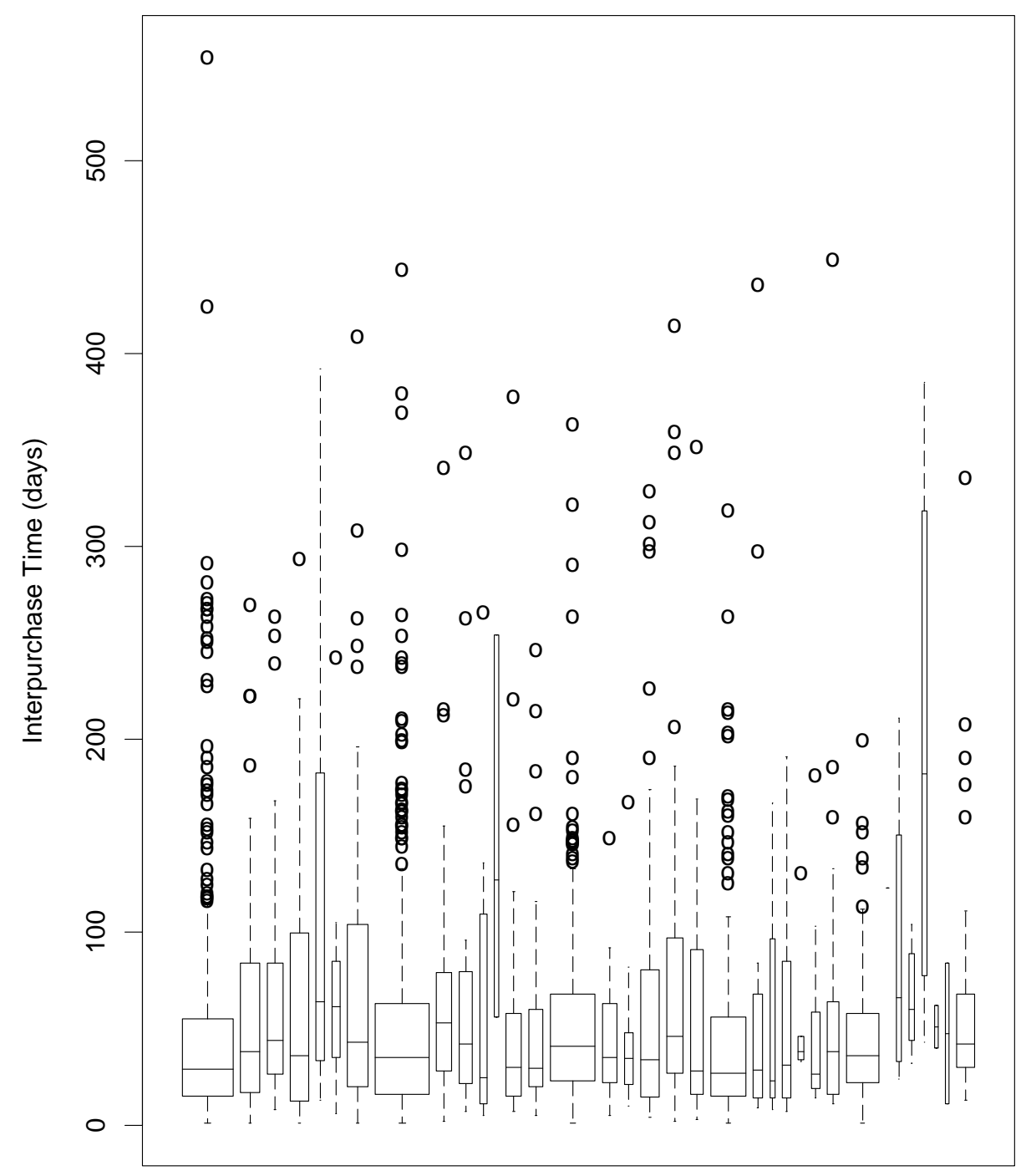

Figure 1: Side by side box plots of interpurchase times for the $6 \times 6$ transitions.

One of the objectives of our study is to investigate the relationship between the pattern of brand switching and inter-purchase times. A comparison of the empirical distributions for the inter-purchase times between transitions should provide some insights. Figure 1 exhibits multiple box plots arranged in order from left to right, where each plot summarizes the inter-purchase times for the 


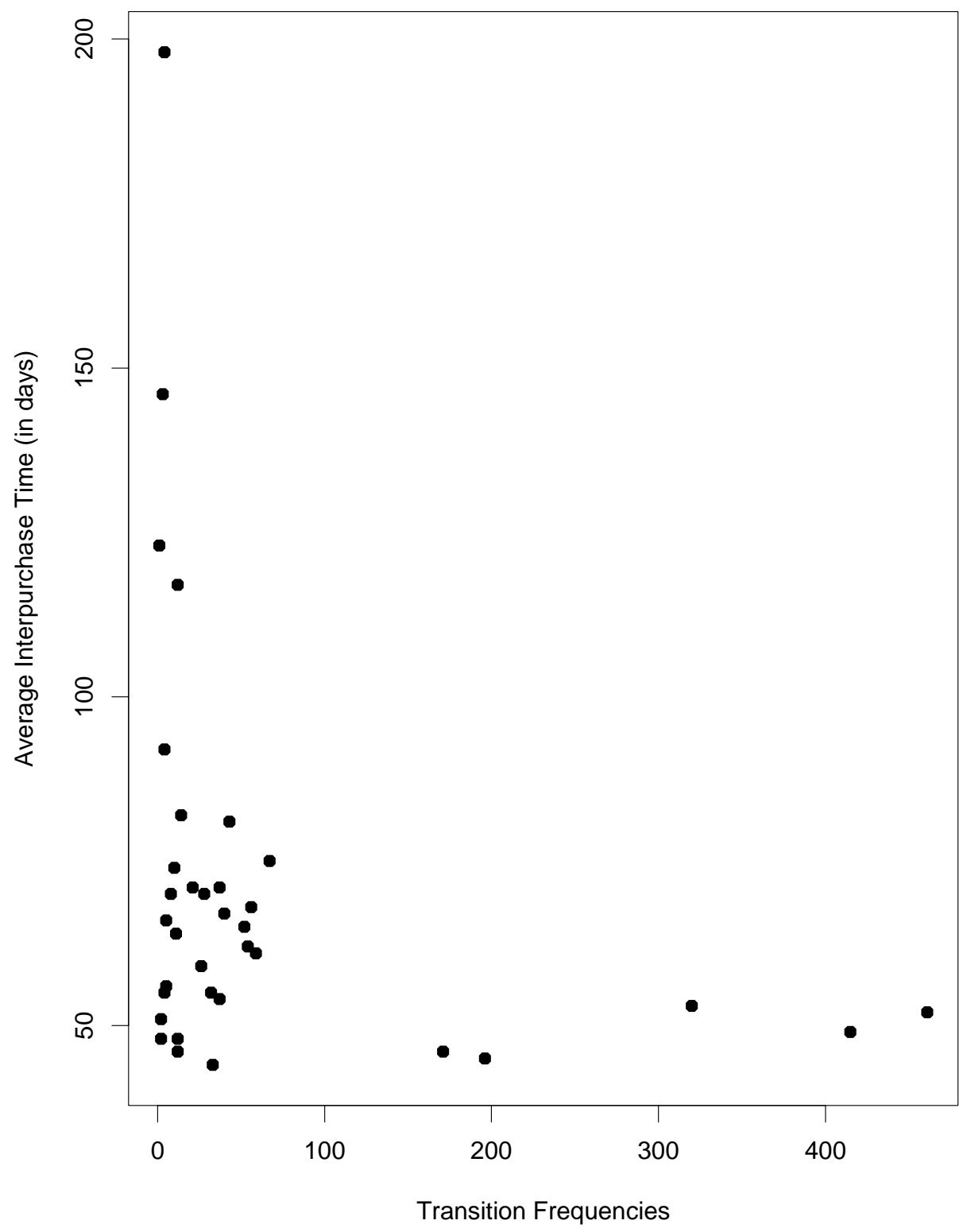

Figure 2: Average interpurchase time vs transition frequencies

$1 \rightarrow 1,1 \rightarrow 2, \ldots, 6 \rightarrow 6$ transitions except that the $3 \rightarrow 6$ transition is missing (because of no data). An interesting finding is that the larger the frequency is, the shorter the inter-purchase time is. This is further confirmed by Figure 
2 , where the average inter-purchase times are plotted against the magnitude of the frequencies for each transition. A very strong curvilinear relationship is exhibited in Figure 2. This figure strongly supports the need to model the bivariate outcomes jointly. Any marginal analysis will not be able to uncover this interesting feature.

The choice-specific variables collected in the sample include the prices (PRICE), features advertisement (FEATURE), and special displays (DISPLAY) of the six bands. The later two are both binary variables, with 1 indicating the presence of feature advertisement or special store display at the time of purchase for each brand. The unit of the price variables is cents per ounce. Since different pack sizes of different brands exist, only the prices of the purchased size are recorded for the competing brands. The chooser-specific variables include household size (HHSIZE), volume of detergent bought last time (STOCK), and non-detergent expenditure this trip (EXPEND). The last variable acts as proxy for "regular" and "fill-in" trips. We calculated the correlations among these variables and found that feature advertisement and special store display for some brands are highly correlated. Particularly, the values of feature advertisement are exactly the same as the values of store display for the brand ALL. Therefore, to avoid collinearity, we drop one of them in the analysis.

According to the proposed model in Section 2, we estimated 6 discrete choice models, each one with a different previous brand, and 36 Cox regression models, each one for a unique transition. The results are reported in Tables 3, 4 and 5 under the name of choice models and duration models, respectively. We report the results without the special display (DISPLAY) variable in both cases. In choice models, only household size from the chooser-specific variables was found to be significant. So, in the final analysis, we dropped the stock and expenditure variables. In duration models, preliminary analysis incorporating the price and feature variables of the competing brands showed them to be statistically insignificant for most of the cases. Therefore they were not included in the final estimation. Only price and feature of the brand purchased are included. Because of limited sample sizes, some of the duration models return no results or unreliable results. In these cases, we use dashes to represent the estimates.

In the following, we discuss the results from Tables 3,4 and 5 in more detail.

\subsection{Choice models}

Examining the effect of choice-specific variables (PRICE and FEATURE) on the probability of purchasing a particular brand, we see that all estimated

coefficients are of the expected signs and all of them are significant at the 0.05 level. The estimated coefficients can be interpreted as in the usual McFadden 
Table 3: Parameter estimates for choice models

\begin{tabular}{lcccccc}
\hline & \multicolumn{5}{c}{ Previous } & Brand \\
\cline { 2 - 7 } Variables & TIDE & WISK & ERAPLUS & SURF & SOLO & ALL \\
\hline INT (T) & & 0.195 & $-2.643^{a}$ & $1.384^{a}$ & $-2.566^{a}$ & -0.891 \\
& & $(0.387)$ & $(0.521)$ & $(0.486)$ & $(0.859)$ & $(2.572)$ \\
INT (W) & $-3.687^{a}$ & & $-3.075^{a}$ & 0.052 & $-4.483^{a}$ & $-3.176^{c}$ \\
& $(0.450)$ & & $(0.522)$ & $(0.513)$ & $(1.254)$ & $(1.781)$ \\
INT (E) & $-3.052^{a}$ & -0.485 & & -0.067 & $-3.906^{a}$ & -3.642 \\
& $(0.539)$ & $(0.495)$ & & $(0.609)$ & $(0.917)$ & $(2.808)$ \\
INT (Su) & $-2.918^{a}$ & $-1.116^{b}$ & $-3.886^{a}$ & & $-5.787^{a}$ & 1.299 \\
& $(0.498)$ & $(0.536)$ & $(0.584)$ & & $(0.957)$ & $(1.745)$ \\
INT (So) & $-2.517^{a}$ & -1.158 & $-2.625^{a}$ & $-2.796^{a}$ & & 0.744 \\
& $(0.771)$ & $(0.884)$ & $(0.730)$ & $(0.916)$ & & $(2.268)$ \\
INT (A) & $-4.394^{a}$ & $-4.000^{a}$ & na & -0.870 & na & \\
& $(0.866)$ & $(1.327)$ & na & $(1.256)$ & na & \\
HHSIZE (T) & & -0.189 & 0.048 & $-0.555^{a}$ & -0.091 & 0.161 \\
& & $(0.116)$ & $(0.131)$ & $(0.140)$ & $(0.297)$ & $(0.896)$ \\
HHSIZE (W) & -0.125 & & $-0.312^{a}$ & $-0.867^{a}$ & -0.179 & 0.466 \\
& $(0.124)$ & & $(0.145)$ & $(0.176)$ & $(0.439)$ & $(0.592)$ \\
HHSIZE (E) & -0.197 & -0.099 & & -0.213 & $0.457^{c}$ & $1.525^{b}$ \\
& $(0.149)$ & $(0.151)$ & & $(0.155)$ & $(0.253)$ & $(0.744)$ \\
HHSIZE (Su) & -0.162 & $-0.481^{b}$ & 0.076 & & $0.829^{a}$ & -1.124 \\
& $(0.148)$ & $(0.198)$ & $(0.140)$ & & $(0.235)$ & $(0.984)$ \\
HHSIZE (So) & -0.441 & -0.432 & -0.254 & 0.278 & & -0.780 \\
& $(0.269)$ & $(0.302)$ & $(0.216)$ & $(0.202)$ & & $(1.116)$ \\
HHSIZE (A) & $-0.860^{b}$ & -0.826 & na & $-2.011^{a}$ & na & \\
PRICE & $(0.349)$ & $(0.589)$ & na & $(0.741)$ & na & \\
FEATURE & $-1.424^{a}$ & $-1.348^{a}$ & $-1.168^{a}$ & $-1.418^{a}$ & $-0.970^{a}$ & $-1.929^{a}$ \\
& $(0.117)$ & $(0.116)$ & $(0.127)$ & $(0.134)$ & $(0.187)$ & $(0.507)$ \\
& $1.739^{a}$ & $1.164^{a}$ & $1.348^{a}$ & $1.775^{a}$ & $1.046^{b}$ & $2.586^{a}$ \\
& $(0.259)$ & $(0.224)$ & $(0.278)$ & $(0.318)$ & $(0.487)$ & $(0.894)$ \\
\hline
\end{tabular}

The standard errors are in parentheses; $\mathrm{T}=\mathrm{TIDE}, \mathrm{W}=\mathrm{WISK}, \mathrm{E}=\mathrm{ERAPLUS}$, $\mathrm{Su}=\mathrm{SURF}$, So $=\mathrm{SOLO}, \mathrm{A}=$ all; ${ }^{a}$ significant at 0.01 level, ${ }^{b}$ significant at 0.05 level, ${ }^{c}$ significant at 0.10 level.

model. For example, reading from column 2 of Table 3 for the brand Tide, we see that an 1-cent increase in the difference between the price of any particular brand and another brand decreases the odds of buying this brand by $24 \%$ (exp($1.424)$ ); and a brand being on feature advertisement (versus a brand without the advertisement) increases the odds of the buying this brand by a factor of 5.7 
Table 4: Parameter estimates for duration models (1)

\begin{tabular}{|c|c|c|c|c|c|c|}
\hline & $\mathrm{T} \rightarrow \mathrm{T}(415)$ & $\mathrm{T} \rightarrow \mathrm{W}(59)$ & $\mathrm{T} \rightarrow \mathrm{E}(40)$ & $\mathrm{T} \rightarrow \mathrm{Su}(52)$ & $\mathrm{T} \rightarrow$ So $(12)$ & $\mathrm{T} \rightarrow \mathrm{A}(10)$ \\
\hline \multirow[t]{2}{*}{ HHSIZE } & $0.093^{a}$ & -0.064 & $0.390^{b}$ & 0.062 & $6.597^{b}$ & -1.183 \\
\hline & $(0.04)$ & $(0.093)$ & $(0.162)$ & $(0.117)$ & $(2.939)$ & $(1.132)$ \\
\hline \multirow[t]{2}{*}{ STOCK } & $-0.007^{a}$ & -0.003 & 0.003 & -0.008 & 0.051 & $0.077^{b}$ \\
\hline & $(0.002)$ & $(0.005)$ & $(0.006)$ & $(0.006)$ & $(0.038)$ & $(0.034)$ \\
\hline \multirow{2}{*}{ EXPEND } & 0.001 & 0.008 & -0.009 & -0.006 & $-0.030^{b}$ & $0.189^{b}$ \\
\hline & $(0.001)$ & $(0.005)$ & $(0.005)$ & $(0.006)$ & $(0.014)$ & $(0.089)$ \\
\hline \multirow{2}{*}{ PRICE } & $0.002^{a}$ & -0.000 & 0.002 & -0.001 & -0.012 & 0.867 \\
\hline & $(0.001)$ & $(0.002)$ & $(0.002)$ & $(0.002)$ & $(0.008)$ & $(0.909)$ \\
\hline \multirow[t]{2}{*}{ FEATURE } & 0.171 & 0.440 & -0.088 & -0.253 & $-8.564^{b}$ & - \\
\hline & $(0.131)$ & $(0.353)$ & $(0.564)$ & $(0.367)$ & (3.991) & - \\
\hline \multirow{3}{*}{ HHSIZE } & $\mathrm{W} \rightarrow \mathrm{T}(67)$ & $\mathrm{W} \rightarrow \mathrm{W}(461)$ & $\mathrm{W} \rightarrow \mathrm{E}(37)$ & $\mathrm{W} \rightarrow \mathrm{Su}(28)$ & $\mathrm{W} \rightarrow$ So $(8)$ & $\mathrm{W} \rightarrow \mathrm{A}$ \\
\hline & -0.120 & $0.258^{a}$ & 0.216 & $0.652^{b}$ & $3.161^{c}$ & - \\
\hline & $(0.104)$ & $(0.038)$ & $(0.163)$ & $(0.300)$ & $(1.772)$ & - \\
\hline \multirow[t]{2}{*}{ STOCK } & -0.003 & -0.001 & -0.007 & $-0.014^{c}$ & 0.053 & - \\
\hline & $(0.004)$ & $(0.001)$ & $(0.008)$ & $(0.008)$ & $(0.052)$ & - \\
\hline \multirow[t]{2}{*}{ EXPEND } & 0.001 & -0.001 & -0.002 & -0.006 & -0.098 & - \\
\hline & $(0.004)$ & $(0.001)$ & $(0.004)$ & $(0.008)$ & $(0.078)$ & - \\
\hline \multirow[t]{2}{*}{ PRICE } & -0.000 & 0.001 & -0.001 & $0.008^{a}$ & $0.034^{c}$ & - \\
\hline & $(0.002)$ & $(0.001)$ & $(0.003)$ & $(0.003)$ & $(0.020)$ & - \\
\hline \multirow[t]{2}{*}{ FEATURE } & -0.113 & 0.135 & 0.171 & 0.265 & 4.259 & - \\
\hline & $(0.288)$ & $(0.110)$ & $(0.465)$ & $(0.571)$ & $(3.967)$ & - \\
\hline \multirow{3}{*}{ HHSIZE } & $\mathrm{E} \rightarrow \mathrm{T}(37)$ & $\mathrm{E} \rightarrow \mathrm{W}(32)$ & $\mathrm{E} \rightarrow \mathrm{E}(320)$ & $\mathrm{E} \rightarrow \mathrm{Su}(33)$ & $\mathrm{E} \rightarrow$ So $(12)$ & $\mathrm{E} \rightarrow \mathrm{A}(0)$ \\
\hline & -0.104 & -0.224 & $0.072^{c}$ & 0.189 & $0.793^{c}$ & - \\
\hline & $(0.165)$ & $(0.159)$ & $(0.038)$ & $(0.147)$ & $(0.412)$ & - \\
\hline \multirow[t]{2}{*}{ STOCK } & $-0.011^{b}$ & 0.004 & $-0.006^{a}$ & -0.010 & 0.041 & - \\
\hline & $(0.006)$ & $(0.006)$ & $(0.002)$ & $(0.007)$ & $(0.025)$ & - \\
\hline \multirow[t]{2}{*}{ EXPEND } & 0.005 & 0.005 & 0.003 & -0.003 & $-0.033^{c}$ & - \\
\hline & $(0.005)$ & $(0.008)$ & $(0.002)$ & $(0.011)$ & $(0.019)$ & - \\
\hline \multirow[t]{2}{*}{ PRICE } & -0.000 & -0.002 & $0.002^{a}$ & -0.000 & $0.011^{b}$ & - \\
\hline & $(0.003)$ & $(0.003)$ & $(0.001)$ & $(0.001)$ & $(0.005)$ & - \\
\hline \multirow[t]{2}{*}{ FEATURE } & 0.191 & 0.258 & -0.061 & 0.130 & 1.709 & - \\
\hline & $(0.489)$ & $(0.414)$ & $(0.191)$ & $(0.570)$ & $(1.381)$ & - \\
\hline
\end{tabular}

$(\exp (1.739))$. The intercepts represent the intrinsic preference for the corresponding brands relative to the base brand. In each of the six models, we make the previous brand the base brand. From Table 3, we see that most of the estimated intercepts are negative, and among those intercepts that are significantly different from zero, all but one are negative. This reflects the feature of the transition pattern: consumers are less likely to change from one brand to another. Based on the intercept estimates, for each previous brand, we can order the brands from the most favored to the least favored. For example, we see that for consumers with a previous purchase of Tide, the first choice is TIDE, the second choice is SOLO, and then SURF, ERAPLUS, WISK, and the last ALL.

Also from Table 3, we see that the chooser-specific variable, household size, plays an non-negligible role in brand choice decision. The effects of household size on the choice of different brands are mixed. For example, for those who bought brand SURF last time, one more household member decreases the odds of 
Table 5: Parameter Estimates for Duration Models (2)

\begin{tabular}{|c|c|c|c|c|c|c|}
\hline & $\mathrm{Su} \rightarrow \mathrm{T}(56)$ & $\mathrm{Su} \rightarrow \mathrm{W}(43)$ & $\mathrm{Su} \rightarrow \mathrm{E}(26)$ & $\mathrm{Su} \rightarrow \mathrm{Su}(196)$ & $\mathrm{Su} \rightarrow \mathrm{So}(14)$ & $\mathrm{Su} \rightarrow \mathrm{A}(4)$ \\
\hline \multirow[t]{2}{*}{ HHSIZE } & $0.218^{c}$ & 0.145 & $0.301^{b}$ & $0.133^{a}$ & 5.811 & - \\
\hline & $(0.119)$ & $(0.151)$ & $(0.140)$ & $(0.043)$ & $(549.8)$ & - \\
\hline \multirow[t]{2}{*}{ STOCK } & $-0.011^{c}$ & 0.002 & -0.006 & $-0.006^{b}$ & $0.019^{c}$ & - \\
\hline & $(0.006)$ & $(0.006)$ & $(0.012)$ & $(0.003)$ & $(0.011)$ & - \\
\hline \multirow[t]{2}{*}{ EXPEND } & $-0.014^{c}$ & 0.006 & -0.000 & 0.004 & $0.029^{a}$ & - \\
\hline & $(0.008)$ & $(0.006)$ & $(0.002)$ & $(0.003)$ & $(0.015)$ & - \\
\hline \multirow[t]{2}{*}{ PRICE } & $0.006^{a}$ & -0.001 & -0.001 & 0.001 & -0.004 & - \\
\hline & $(0.002)$ & $(0.002)$ & $(0.002)$ & $(0.001)$ & $(0.006)$ & - \\
\hline \multirow[t]{2}{*}{ FEATURE } & $-0.809^{b}$ & -0.555 & -0.239 & -0.049 & - & - \\
\hline & $(0.350)$ & $(0.382)$ & $(0.579)$ & $(0.241)$ & - & - \\
\hline \multirow{3}{*}{ HHSIZE } & $\mathrm{So} \rightarrow \mathrm{T}(11)$ & $\mathrm{So} \rightarrow \mathrm{W}(5)$ & $\mathrm{So} \rightarrow \mathrm{E}(12)$ & $\mathrm{So} \rightarrow \mathrm{Su}(21)$ & So $\rightarrow$ So $(171)$ & $\mathrm{So} \rightarrow \mathrm{A}(1)$ \\
\hline & -0.243 & - & 0.305 & 0.211 & -0.010 & - \\
\hline & $(0.429)$ & - & $(0.401)$ & $(0.187)$ & $(0.076)$ & - \\
\hline \multirow[t]{2}{*}{ STOCK } & -0.008 & - & 0.012 & 0.001 & -0.001 & - \\
\hline & $(0.030)$ & - & $(0.015)$ & $(0.008)$ & $(0.002)$ & - \\
\hline \multirow[t]{2}{*}{ EXPEND } & -0.001 & - & -0.029 & $0.007^{c}$ & $0.003^{c}$ & - \\
\hline & $(0.003)$ & - & $(0.024)$ & $(0.004)$ & $(0.001)$ & - \\
\hline \multirow[t]{2}{*}{ PRICE } & 0.010 & - & -0.013 & -0.001 & $0.003^{b}$ & - \\
\hline & $(0.009)$ & - & $(0.009)$ & $(0.002)$ & $(0.001)$ & - \\
\hline \multirow[t]{2}{*}{ FEATURE } & 1.659 & - & - & -0.406 & 0.281 & - \\
\hline & (1.138) & - & - & $(0.655)$ & $(0.377)$ & - \\
\hline \multirow{3}{*}{ HHSIZE } & $\mathrm{A} \rightarrow \mathrm{T}(4)$ & $\mathrm{A} \rightarrow \mathrm{W}(5)$ & $\mathrm{A} \rightarrow \mathrm{E}(4)$ & $\mathrm{A} \rightarrow \mathrm{Su}(2)$ & $\mathrm{A} \rightarrow$ So $(2)$ & $\mathrm{A} \rightarrow \mathrm{A}(54)$ \\
\hline & - & - & - & - & - & $0.352^{b}$ \\
\hline & - & - & - & - & - & $(0.162)$ \\
\hline \multirow[t]{2}{*}{ STOCK } & - & - & - & - & - & -0.005 \\
\hline & - & - & - & - & - & $(0.006)$ \\
\hline \multirow[t]{2}{*}{ EXPEN } & - & - & - & - & - & 0.001 \\
\hline & - & - & - & - & - & $(0.005)$ \\
\hline \multirow[t]{2}{*}{ PRICE } & - & - & - & - & - & -0.180 \\
\hline & - & - & - & - & - & $(0.296)$ \\
\hline \multirow[t]{2}{*}{ FEATURE } & - & - & - & - & - & - \\
\hline & - & - & - & - & - & - \\
\hline
\end{tabular}

purchasing Tide by $57 \%$, while for those who bought SOLO last time, one more family member increases the odds of purchasing ERAPLUS by about 1.6 times.

Our findings here from the choice models agree with previous findings about brand choice (Jain et al. 1994) in the sense of the choice-specific variables and the consumer intrinsic preference. The real implication of the effect of household size needs more investigation.

\subsection{Duration models}

Tables 4 and 5 report the estimated coefficients of chooser-specific variables and choice-specific variables from the 36 Cox regression models. The number inside the parentheses after the name of transitions are the sample size for that transition. In some models, there is not enough data to estimate the coefficients reliably. In some other models, one or more variables might take the same value, 


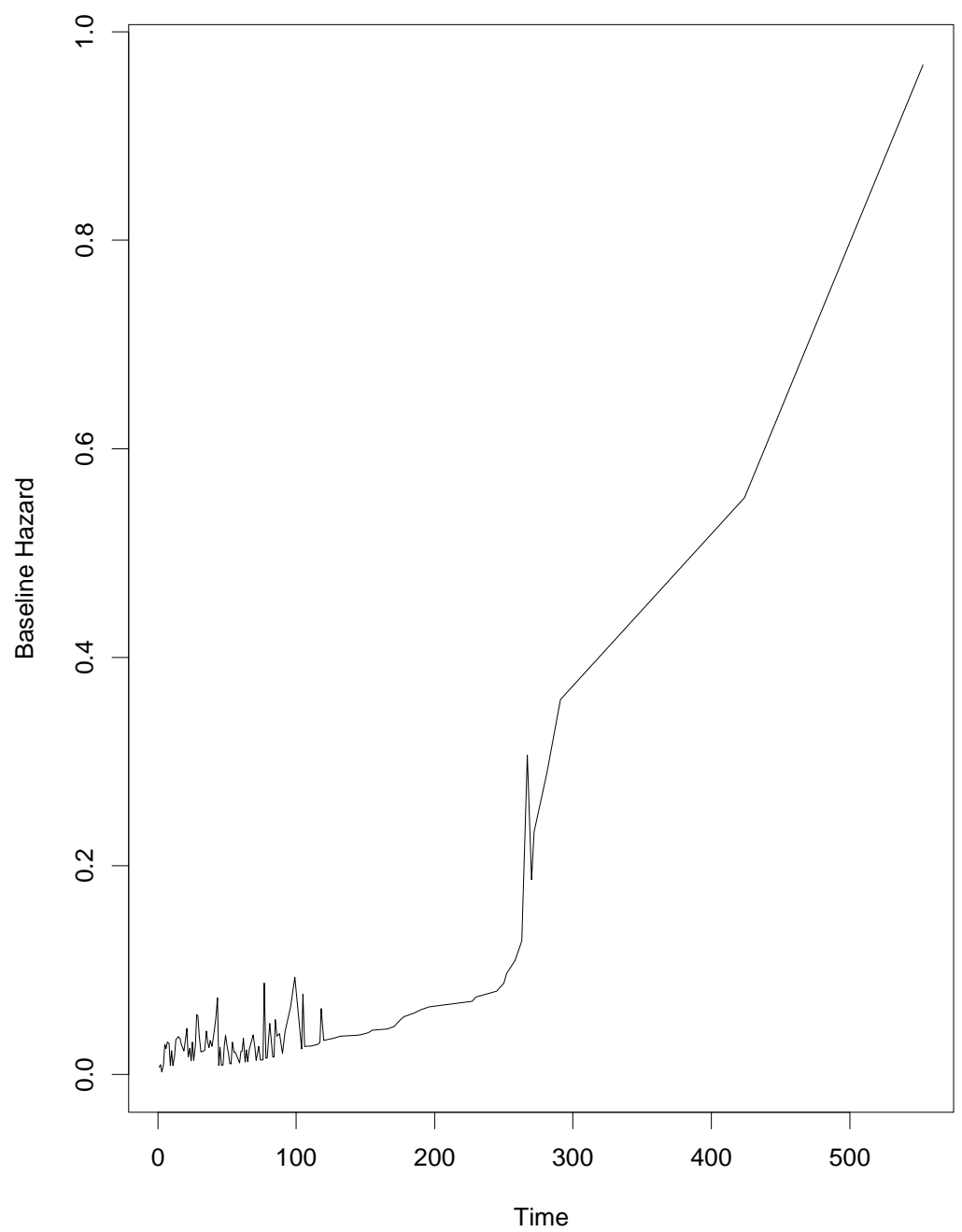

Figure 3: Estimated baseline hazard function for transition TIDE $\rightarrow$ TIDE

thus making the coefficients of these variables unidentifiable. In these cases, we use dashes to represent the estimates.

From Tables 4 and 5, we see that most of the estimated coefficients for the chooser-specific variables are of the expected signs, especially for the ones that are significant. Household size (HHSIZE) generally has positive estimated coefficients, suggesting that an increase of household size will likely increase the rate (hazard) of making a purchase. On the other hand, volume purchased at previous occasion (STOCK) generally has negative signs which says that the more 
you bought last time (the more you have as stock) the less the rate of making the next purchase. The effect of non-detergent expenditure (EXPEND) on the rate of making the next purchase is not that significant in general, and for those cases where it is significant, the effects are mixed.

Turning to the choice-specific variables, we note that feature advertisement (FEATURE) is generally not significant. Price is significant for some of the models and has positive coefficients. This implies that as the price of the brand goes up, the rate of making the purchase increases. For example, when the transition is from WISK to SURF, one cent increase of the SURF's price increases the odds of purchasing SURF by 1.008. The magnitude of the changes in odds is small. Nevertheless, this finding of the price effect on the timing decision is an interesting departure from some previous findings (see Vilcassim and Jain 1991 and Chintagunta and Prasad 1998). In those studies, the price variable usually has the opposite effect. However, our finding does make some intuitive sense: as the price of the brand goes up, consumers might be anticipating further price increases which encourages consumers to make the purchase sooner rather than later.

In Figure 3, we plot the estimated baseline hazard function for transition times from TIDE to TIDE. We see that the baseline hazard is nonmonotonic. Particularly, it fluctuates frequently with many peak and valleys, and with piecewise local trends. This feature of the baseline hazard precludes the use of the Box-Cox formulation for the baseline hazard.

We also conducted the analysis without the second assumption in (2.4) where we include the previous inter-purchase time (PREDUR) in the covariates for each duration model in a transition. The analysis revealed that the predictor (PREDUR) is significant at the 0.05 level only in a few models. The price when significant still has the same positive sign as in Tables 4 and 5 . The results revealed very similar qualitative interpretation as in Tables 4 and 5. Therefore, the analysis is omitted here.

\section{Summary and Discussions}

In this paper, we developed a new method to model jointly the inter-purchase time and brand choice. Our model accounts for the dependence structure across successive purchases from the same household via a first-order Markov property. For the decomposition of the joint model, we found that conditioning on the brand choice variable has more advantages than the dynamic McFadden model where the conditioning is done on the inter-purchase time. It allows us to fit the discrete choice model and duration model separately. In addition, it also allows us to fit different distributions for the duration times between different transitions and different multinomial logit models for different previous brand choices. Best 
of all, all these fittings can be easily done with commonly used software that includes SAS or S-Plus.

We applied our model to scanner data of detergent purchase. We found that among choice-specific variables, price and feature advertisement are significant factors for consumer's choice decisions. Household size, as a chooser-specific variable, is also important in the choice decision process. We also found that price is a significant factor in the timing decision.

Data set similar to the one used in this paper also appear in other fields. For example, in labor economics, data are collected on when a person changes labor status among the employed, unemployed, and out of labor force status (cf. Lancaster 1990, and Heckman and Singer 1985). In sociology, event history data on marriage, separation, and divorce may be collected (cf. Yamaguchi 1991). In medicine, life history data are collected to study relapse-remitting processes (cf. Anderson, Borgan, Gill, and Keiding 1993). In these situations, our proposed method can be utilized with slight modifications. For instance, we may want to modify our multinomial logit model to accommodate the no-repetition transition. Our model can be made more parsimonious by assuming some of the transitions share the same model. For example, we might assume $\boldsymbol{\beta}^{*(l)}=\boldsymbol{\beta}^{*(m)}$ if we believe the switching patterns are quite similar between the last purchase choices $l$ or $m$. Similarly, we can make equal coefficients assumptions for some of the parameters in the Cox model.

Our method cannot yield stable results for small sample size. The above discussion on collapsing models would help the sample size problem. Furthermore, we can employ the techniques used in small domain estimation to borrow strength from neighboring categories to treat small sample-size problems. We have ignored the incomplete observations at the two end points of the panel data for each household. The study can be improved by adding the incomplete observations. For the beginning of the monitoring period, we can impute the state space variable at time 0 . That is, we impute the brand choice for the last purchase before the monitoring period. Various imputation schemes can be explored. It would be interesting to study the effects of the imputation schemes. For the end of the study, we can include right-censored data for the time between the last purchase and the ending monitoring period and imputed ending states. The censored data part can be easily done in each of the 36 Cox regression models.

We have adopted a first-order Markov assumption in our derivation of the likelihood. This assumption can be relaxed to incorporate higher order (cf. Diggle, Liang, and Zeger 1994, p. 195). Although it increases the complexity of the analysis, it may be needed when the $\mathrm{AR}(1)$ assumption is known to be inadequate.

We have made two assumptions in the conditioning decomposition of our 
likelihood. First, we assume $f\left(y_{i j} \mid t_{i j}, y_{i, j-1}\right)=f\left(y_{i j} \mid y_{i, j-1}\right)$, then we use the discrete choice model with the explanatory variables from the $j$ th purchase of consumer $i$ to model this transition state space model. With time varying covariates, it would be worthwhile to consider models with explanatory variable from the $(j-1)$ th purchase of consumer $i$. Given that this transition model should depend on the inter-purchase time, we can relax our first assumption by allowing the inter-purchase time $t_{i j}$ as part of the explanatory variable. On the second assumption, we assume $f\left(t_{i j} \mid y_{i j}, t_{i, j-1}, y_{i, j-1}\right)=f\left(t_{i j} \mid y_{i j}, y_{i, j-1}\right)$. This assumption can be relaxed by adding $t_{i, j-1}$ as a covariate in the Cox regression model. Our analysis (omitted here) reveals that the dependence on the previous inter-purchase time for the scanner data is not very significant.

On the duration modeling, in addition to the Cox regression model, we can also consider the log linear model, the additive hazards regression model, or the generalized additive hazards models (Hastie and Tibshirani, 1990). Unobserved hetorogeniety among households would often affect the analysis (cf. Jain, Vilcassim, and Chintagunta 1994, Chintagunta and Prasad 1998). It would be worthwhile to incorporate a random-effects specification (frailty) into our model and compare the two results.

We have assumed the state space remained unchanged during study. Often, a new product is introduced into the market, or a product is phased out. It would, therefore, be worthwhile to consider dynamic models and change-point models for this kind of situation.

\section{References}

Agresti, A. (1990). Categorical Data Analysis. John Wiley and Sons.

Andersen, P. K., Borgan, O., Gill, R. D., and Keiding, N. (1993). Statistical Models Based on Counting Processes. Springer-Verlag.

Breslow, N. E. (1974). Covariance Analysis of Censored Survival Data. Biometrics 30, 89-99.

Chintagunta, P. K., and Prasad, A. R. (1998). An Empirical Investigation of the 'Dynamic McFadden' Model of Purchase Timing and Brand Choice: Implications for Market Structure. Journal of Business and Economic Statistics 16, 2-12.

Cox, D. R. (1972). Regression Models and Life-tables (with discussion). Journal of the Royal Statistical Society, Series B 34, 187-220.

Diggle, P. J., Liang, K., and Zeger, S. L. (1994). Analysis of Longitudinal Data. Oxford Science Publications.

Flinn, C., and Heckman, J. (1982). Models for the Analysis of Labor Force Dynamics. In Advances in Econometrics, 1.R (Edited by Bassmann and G. Rhodes). JAI Press. 
Flinn, C., and Heckman, J. (1983). The Likelihood Function for the MultistateMultiepisode Model in 'Models for the Analysis of Labor Force Dynamics'. In Advances in Econometrics, 3.R (Edited by Bassmann and G. Rhodes). JAI Press.

Gönül, F., and Srinivasan, K. (1993). Consumer Purchase Behavior in a Frequently Bought Product Category: Estimation Issues and Managerial Insights from a Hazard Function Model with Heterogeneity. Journal of the American Statistical Association $\mathbf{8 8}, 1219-1227$.

Hastie, T. J., and Tibshirani, R.J. (1990). Generalized Additive Models. Chapman and Hall.

Heckman, J., and Singer, B. (1984). A Method for Minimizing the Impact of Distributional Assumptions in Econometric Models for Duration Data. Econometrica 52, 271-320.

Heckman, J., and Singer, B. (1985). Longitudinal Analysis of Labor Market Data. Cambridge University Press.

Hosmer, D. W., and Lemeshow, S. (1989). Applied Logistic Regression, John Wiley and Sons.

Jain, D. C, and Vilcassim, N. J. (1991). Investigating Household Purchase Timing Decisions: A Conditional Hazard Function Approach. Marketing Science 10, 123.

Jain, D. C., Vilcassim, N. J., and Chintagunta, P. K. (1994). A Random-Coefficients Logit Brand-Choice Model Applied to Panel Data. Journal of Business and Economic Statistics 12, 317-328.

Kalbfleisch, J. D., and Prentice, R. L. (1980). The Statistical Analysis of Failure Time Data. John Wiley and Sons.

Klein, J. P., and Moeschberger, M. L. (1997). Survival Analysis Techniques for Censored and Truncated Data. Springer.

Lancaster, T. (1990). The Econometric Analysis of Transition Data. Cambridge University Press.

Lawless, J. F., and Fong, D. Y. T. (1999). State Duration Models in Clinical and Observational Studies. Statistics in Medicine 18, 2365-2376.

McCullagh, P., and Nelder, J. A. (1989). Generalized Linear Models, (2nd ed). Chapman and Hall.

McFadden, D. (1974). Conditional Logit Analysis of Qualitative Choice Behavior. In Frontiers of Econometrics (Edited by P. Zarembka), 105-142. Academic Press.

Prentice, R. L., Kalbfleisch, J. D., Peterson, A.V. Jr., Flounoy, N., Farewell, V. T., and Breslow, N. E. (1978). The Analysis of Failure Times in the Presence of Competing Risks. Biometrics 34, 541-554.

SAS Institute Inc. (1995). Logistic Regression Examples Using the SAS System, Version 6 , (1st ed.) SAS Institute. 
Vilcassim, N. J., and Jain, D. C. (1991). Modeling Purchase-Timing and BrandSwitching Behavior Incorporating Explanatory Variables and Unobserved Heterogeneity. Journal of Marketing Research 28, 29-41.

Yamaguchi, K. (1991). Event History Analysis. SAGE Publications.

Received October 3, 2002; accepted April 1, 2003.

Lynn Kuo

Department of Statistics, U-4120

University of Connecticut

Storrs, CT 06269 USA

lynn@stat.uconn.edu

Zhen Chen

Department of Biostatistics and Epidemiology

University of Pennsylvania

423 Guardian Drive - 625 Blockley Hall

Philadelphia, PA 19104, USA

zchen@cceb.upenn.edu 